\title{
常绿阔叶树种栲树开花物候动态及花的空间配置
}

\author{
陈 波 ${ }^{1}$ 达良俊 ${ }^{2}$ 宋永昌 $^{2}$ \\ （1 杭州师范学院生命科学学院 杭州 310036）（2 华东师范大学环境科学系, 上海 200062)
}

摘 要 基于定株观测和随机枝取样法, 对浙江天童常绿阔叶林内栲树 (Castanopsis fargesii) 的开花物候动态及其雌 花、雄花的空间配置进行了研究。结果表明: 在栲树的生殖枝上, 并非所有的芽都分化、萌发生成花序栲树花芽的 分化和发育集中在一级生殖枝上。生殖枝上花芽的分化与该枝的空间位置密切相关。栲树花期明显晚于春季的 展叶期, 与叶片生长时间重叠。盛花期集中于 5 月下旬，约持续 $8 \mathrm{~d}$ 左右, 属于同步发生的花期。栲树雄花序的数 量明显高于雌花序 雄花序约占花序总数的 $77.88 \%$ 雌花序仅占 $22.12 \%$ 。大量雄花和花粉的存在是保证雌花接 受花粉和完成受精的基础。花序在植冠层中的空间配置明显不同:在同一植冠内, 向阳面和背阴面生殖枝上芽萌 发成花序的比率存在明显差异 $(p<0.01)$,阳面生殖枝上顶芽萌发成花序的比率高于阴面生殖枝的比率, 并且, 阳 面的每个生殖枝上平均花序数和雄花数量均高于阴面生殖枝 花序的分化和发育与枝系的生长发育状况有密切关 系。

关键词 浙江天童 栲树 开花物候 花和花序 空间配置

\section{FLOWERING PHENOLOGY AND FLORAL DISTRIBUTION OF CASTANOPSIS FARGESII IN TIANTONG , ZHEJIANG PROVINCE}

\author{
CHEN Bo ${ }^{1}$ DA Liang-Jun ${ }^{2}$ and SONG Yong-Chang ${ }^{2}$ \\ (1 School of Life Sciences , Hangzhou Normal College , Hangzhou 310036 , China) \\ (2 Department of Environmental Science, East China Normal University, Shanghai 200062 , China)
}

\begin{abstract}
Forested areas of eastern China are predominatly evergreen broad-leaved forests which are typically zonal or climatic climax vegetation in subtropical areas, dominated by Castanopsis fargesii Franch. (Fagaceae) a shade-tolerant and long-lived species. The observation and study of its reproductive characteristics in the field still remain poorly understood. Based on the methods of Randomized Branch Sampling and observation of labeled individuals, the flowering phenology , flower number and spatial distribution of Castanopsis fargesii Franch. from the Schimeto-Castanopsietum fargesii Association in the Tiantong National Forest Park (TNFP) were studied in this paper. The results showed that the floral bud flushing and inflorescence elongation of $C$. fargesii emerged at end of April and the trees flowered after leaf emergence. The onset of mass flowering occurred at late May, and its duration within population could last more or less eight days, while the flowering time among individuals experienced less difference. Therefore $C$. fargesii displays synchronous flowering. Given the lower precipitation and gradually increasing temperature during April-May, synchronous flowering and therefore pollination in $C$. fargesii is a beneficial adaptation.

Floral buds commonly located at the first-order branch, which made up $91.92 \%$ of all the reproductive branch, nearly $7.07 \%$ and $1.01 \%$ at the second and the third branch, respectively. There was an apparent difference between the floral bud initiation at the toward-sun reproductive branch and the toward-shade ones within a crown $(p<0.01)$ and the floral bud initiation were $78 \% \pm 5.7 \%$ and $67 \% \pm 5.7 \%$ respectively. Within a crown, there were about $4.89 \pm 1.82$ panicles per reproductive branch at sun-side and $3.08 \pm 2.08$ at shade-side.

The number of male inflorescence of $C$. fargesii is more than that of the female and male inflorescence occupied $77.9 \%$ of the total and female inflorescence $22.1 \%$ respectively. There were $67.9 \pm 16.7$ staminate at one male inflorescence and $26.1 \pm 5.08$ pistillate at one female inflorescence. Furthermore , the flower numbers were male biased during spring censuses and the ratio of staminate to pistillate was about 9.2:1. Mass staminate could produce higher pollen concentration during synchronous period and should increase the pollination efficiency. Therefore flowering in spring exerted great influence on the reproductive output of $C$. fargesii .
\end{abstract} Key words TNFP , Castanopsis fargesii , Flowering phenology , Inflorescence , Distribution 
植物开花物候 (Flowering phenology) 的研究是植 物生殖生态学的一个重要内容 (Whitehead,1983; Willson , 1983 ;Lovett Doust \& Lovett Doust , 1988)。开 花物候包括花芽的形成与发育、始花期、盛花期及持 续时间等过程。从植物种群水平研究开花物候, 是 了解植物种的生殖对策的基础, 同时对群落内优势 物种的生殖物候研究, 有助于认识群落的形成过程 及动态。自 Harper (1977) 提出构件理论以来, 人们 已认识到可以将被子植物的花、果实和种子等作为 生殖构件 (Reproductive module) 看待, 生殖构件在植 冠中的分化、生长和分布及与枝系间关系的研究成 为植物个体和种群水平上生殖生态学研究的一个新 方向 (Lovett Doust \& Lovett Doust, 1988; 祖元刚等, 2000)。

栲树 (Castanopsis fargesii) 是亚热带常绿阔叶林 的常见优势种之一, 以该种植物为优势种的常绿阔 叶林广泛分布于我国的亚热带东部地区。不少学者 对以栲树为建群种或优势种的植物群落进行了较为 详尽的研究(宋永昌等, 1995; 刘智慧, 1990; 钟章成, 1988 ;丁圣彦，1999），但对该区优势物种栲树的开花 物候和生殖构件配置的研究未见报道。本文分析研 究了天童地区的栲树花期物候特征及其花序的空间 配置, 以期深入了解栲树的生殖生态特征和生活史 对策。

\section{1 研究地点和研究方法}

\section{1 研究地点}

研究地点位于浙江宁波天童国家森林公园内, $29^{\circ} 48^{\prime} \mathrm{N}, 121^{\circ} 47^{\prime} \mathrm{E}$, 属温暖湿润的亚热带季风气候 区,全年温和多雨，四季分明。常绿阔叶林是天童国 家森林公园的主要植被类型, 可以划分为木荷-栲树 群丛 (Schimeto-Castanopsietum fargesii Association)和绵 槠-云山青冈群丛 ( Lithocarpeto-Cyclobalanopsietum nubii Association)等, 该地区的植被和自然状况已有详 细报道(宋永昌等,1995) 此处不再赘述。

物候观测在华东师范大学生态研究站后的木荷 一栲树林内进行。海拔 $200 \mathrm{~m}$ 坡度约 $25^{\circ} \sim 30^{\circ}$, 群落 覆盖度 $90 \%$ 左右。乔木层以栲树、木荷 (Schima superba) 为主, 灌木层主要包括连荵茶 (Camellia frater$n a) 、$ 浙江新木姜子 ( Neolitsea aurata)、柃木 ( Eurya japonica)、山矾 (Symplocos sumuntia)、老鼠矢 ( S. stellaris)、 口木 (Loropetalum chinense) 等植物。在群落内 标记观测木, 共观测标记 28 株开花植株, 另选取 5 株较低矮易于攀援的植株固定取样分析。

\section{2 研究方法}

\subsection{1 花序定义}

对壳斗科植物花序的描述和定名较复杂。早 期, Brett(1964) 认为壳斗科植物的花序是一种非常 退化的二歧分枝结构，将它们的花序分别简称为雌 花序或雄花序, 并将 Castanea 属中植物的花序称为 $\square$ 荑花序, 认为分布在较短侧枝上的 $\square$ 荑花序几乎 都是雄花, 而在主枝上发育较晚的口荑花序上一般 是雌花。MacDonald (1979) 也将 Quercus 和 Fagus 两 属植物的花序分别称为雌花序或雄花序。而 Fey 和 Endress (1983)认为除 Fagus 和 Nothofagus 属外, 壳斗 科大多植物的雄花序一般是简单的穗状花序或聚伞 花序, 雌花序一般也是穗状花序或聚伞花序。Kaul (1985) 研究 Quercus 属植物的生殖特征时 将其雌性 花序和雄性花序分别称为雌性穗状花序和雄性口荑 花序。

本文通过野外的实际观测，将栲树的单个雄花 序称为 $\square$ 荑花序, 将单个雌花序直接称为雌花序, 同 时将口荑花序(雄花序)或雌花序组合成的复合花序 称为圆雉花序。经观察, 栲树的圆雉花序主要包括 3 种形式: 1) 整个圆雉花序仅由雄花序组成，2)整个 圆雉花序仅由雌花序构成;3) 圆雉花序上由雄花序 和雌花序共同构成, 其中, 雌花序主要分布在圆雉花 序轴的中、上部, 也有少数是分布在圆雉花序轴的下 部。此外, 还有一种类型, 即口荑花序的上部为雌 花，下部为雄花, 但这种形式极少。

\subsection{2 数据观测}

野外观测记录从 2000 年 3 月至 5 月底进行。 开花节律依 Dafni (1992) 描述的方法进行, 记录、观 测植物的主要开花事件, 即根据开花的时间和数量 变化分为几个不同阶段,主要包括始花、开花(1/4 开花至 $1 / 2$ 开花)、盛花及花谢等阶段。观测、记录 固定样株内标记的枝系上芽的萌动情况, 并记录所 观测枝系在植冠层中的位置。本文将植冠层中可以 接受较好的光照条件的一面称为向阳面, 将植冠层 中较少接受光照的一面称为背阴面。同时也采取随 机枝取样方法 (Maillette ,1987 ;Lehtila et al.,1994)， 即在不同时期从开花的栲树植株上取一定数量的枝 系 统计其上的花数和开花的数量, 并测定生殖构件 (花序、花)的大小。

枝序按 Strahler 法确定 (Borchert \& Slade，1981)， 即在植冠层内, 由外及内确定枝序，外层的第一小枝 为第一级, 两个第一级相遇即为第二级, 两个第二级 相遇后则为第三级，依此类推，如有不同枝级相遇， 
相遇后则取较高的作为枝级。生殖枝上的顶芽标记 为 $\mathrm{T}$, 侧芽由小枝远轴端 (Distal) 向近轴端 (Basal) (即下部) 依次确定序号, 分别称其为第一侧芽、第二 侧芽等。

2000 年栲树雌花序的数量较少, 因此在栲树开 花事件中, 主要以雄花序为主。所有数据作相关统 计分析。

\section{2 结果与分析}

\section{1 栲树花的物候和花序的生长}

栲树的芽在当年秋季形成, 属于鳞芽,有约 10 余片芽鳞保护芽度过温度较低的冬季。在光照条件 较好的生境中 栲树芽的萌动期在 3 月下旬左右, 原 来紧实的芽鳞(芽体萌动前宽约 $0.4 \sim 0.5 \mathrm{~cm}$, 高约 $0.5 \sim 0.6 \mathrm{~cm}$ ) 开始疏松, 芽体明显增大, 此时芽体宽 约 $0.6 \sim 0.8 \mathrm{~cm}$, 高约 $0.7 \sim 1.2 \mathrm{~cm}$ 。随后开始抽枝 并形成叶片。在 4 月初至 4 月底这一期间，从外部 形态特征上很难将花芽与叶芽分开, 这一阶段主要 以新枝和叶的生长为主, 但长势较慢。4 月 $20 \sim 25$ 日进入始花期 栲树花序开始从新枝轴的叶腋内生 出, 此时枝轴长约 $(2.32 \pm 1.09) \mathrm{cm}$, 有若干花序构 成圆雉花序; 因此, 此时通过花序的生成与否可以鉴 定 3 月底 4 月初之间已抽枝展叶的芽是否属于花 芽。栲树花芽的萌发及随后的开花时间晚于当年春 季叶芽的萌发和叶片生长时间, 属于先叶后花。

在 4 月底 (25日左右)，栲树的圆雉花序分化并 进入生长阶段, 其枝轴生长见图 1。在生长初期, 圆 雉花序的枝轴生长速度明显较慢, 至 5 月中旬 (17 日)的生长速率约 $0.4 \mathrm{~cm} \mathrm{~d}^{-1}$ 盛花期时圆雉花序的 生长速率最大, 约 $0.7 \mathrm{~cm} \mathrm{~d} \mathrm{~d}^{-1}$ 。盛花期后, 约有 $90 \%$ 的圆雉花序脱落。

少数花芽在其鳞片还没有完全疏松时, 就有花 序从鳞片中伸出, 但大多数分布于圆雉花序上的 $\square$ 荑花序的发育和生长趋势是 近轴端的花序先生成， 随圆雉花序的生长逐渐在上部开始生成花序, 即分 布于圆雉花序枝轴基部的花序先生成。由于 2000 年雌花数量较少, 故野外观测期间主要以雄花序的 生长观测为主。花序的发育和生长略晚于圆雉花序 轴的生长, 但生长较快 (图 2)。在口荑花序的生长 早期阶段 其上分布的花蕾较为密集。5 月 5 日左 右以后, 随着口荑花序的伸长, 花蕾之间距离逐渐拉 开, 并且花序逐渐挺立, 少有下垂, 这一时期主要是 花蕾的生长阶段。在盛花期间口荑花序的长度最 大 约 $(11.77 \pm 2.54) \mathrm{cm}$,与开花的时间较为吻合。

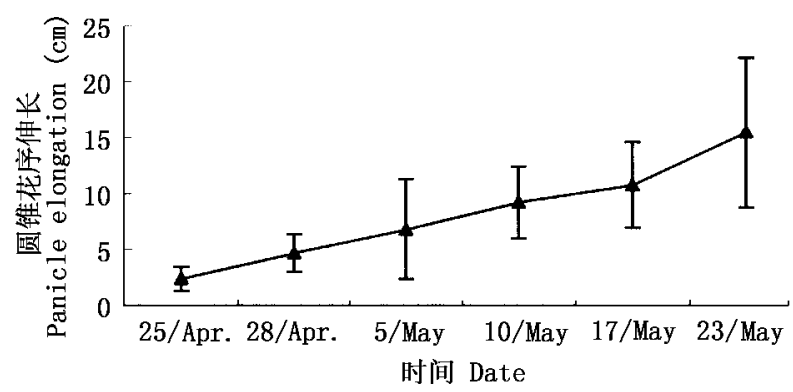

图 1 圆雉花序的生长动态

Fig. 1 Panicle elongation dynamic of Castanopsis fargesii

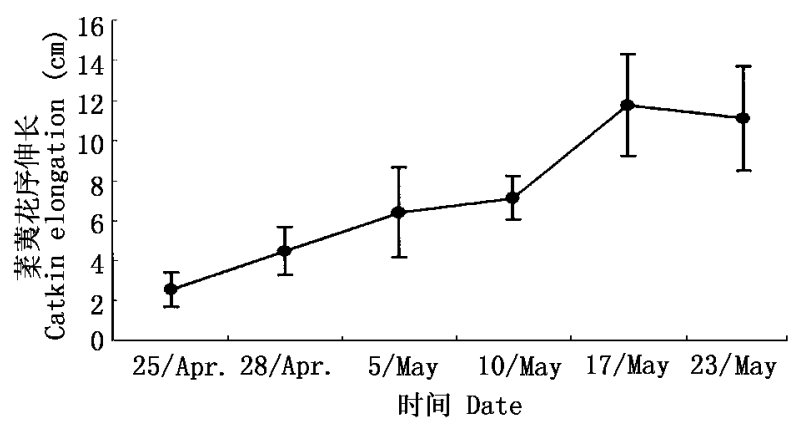

图 2 口荑花序的生长动态

Fig.2 Catkin elongation dynamic of Castanopsis fargesii

口荑花序上的花蕾生长到一定阶段, 发育成熟 并开花。在圆雉花序轴上, 一般是下部的口荑花序 先开，上部的稍晚，这与花序的发育和生长顺序较为 一致，即花序在枝轴上的发育生长顺序与植株体内 营养物质的运输有密切关系, 近轴端先得到营养物 质, 发育生长较早, 而远轴端则较慢。图 3 表示栲树 种群水平的花期动态。5 月 10 日左右, 可以看见少 许雄花开放, 多数雄花花被( 栲树花被数 6) 没有完 全展开，一般仅有 $2 \sim 6$ 根花丝伸出，不及栲树雄花 花丝的一半 栲树雄花花丝一般 12 根; 在盛花期间， 雄花的花被基本全部展开,花丝几乎全部露出。栲 树在种群水平上的花期持续约 $12 \mathrm{~d}$ 左右, 盛花期约 $8 \mathrm{~d}$ 左右, 此时约有 $80 \%$ ～90\% 的雄花序开花。不 同植株的开花顺序并不相同，一般是光线较好的环 境中的个体开花较早, 以栲树为优势的群落地段要 晚 1 2 d。对一株个体而言, 其雄花开放时间也略 有差异 花序形成和花蕾开放均由个体的上部而下。 观察生态站房后的一株开花栲树，花期持续时间约 $6 \mathrm{~d}$ 植株上层的花盛开时，下部的少量的花序仍在 生长中, 在上部大量花朵开花、凋落时，下部的未开 花序也几乎全部凋落, 很少能生长发育到全部开花 阶段。本文在野外观测期间发现 2000 年栲树雌花 序数量较少, 在 5 月中旬 (17 日) 观测到雌花序轴的 平均长约 $(4.5 \pm 1.04) \mathrm{cm}$, 每个雌花序轴上平均有 雌花蕾 $(26.10 \pm 5.08)$ 个。此时每个雌花序轴上约 
有 $22.6 \%$ 雌花开花。5 月下旬(23 24 日)的调查统 计表明, 每个雌花序轴上有 $86.1 \%$ 的雌花开花, 雌 花序长约 $(11.86 \pm 3.42) \mathrm{cm}$, 其生长速率约 $1.2 \mathrm{~cm}$ $\mathrm{d}^{-1}$,比雄花序的生长速率快。

与花的发育和生长时间相比, 栲树花谢和凋落 时间较快。在盛花期, 就有 $\square$ 荑花序或单朵小花凋 落，因此，栲树圆雉花序上的花朵不能达到 $100 \%$ 的 开花程度, 经统计最大的开花率为 $96 \%$ 。盛花期 后, 花谢更快, 2 4 d 基本落完, 如有风、雨等因素, 则凋落更快。4 月 23 日在栲树林内调查发现, 花的 调落量很大, 主要是雄性口荑花序, 从 58 个 $\mathrm{m}^{-2}$ 到 最多的 256 个 $\mathrm{m}^{-2}$, 平均约 $131.9 \pm 53.9$ 个 $\mathrm{m}^{-2}$ 。

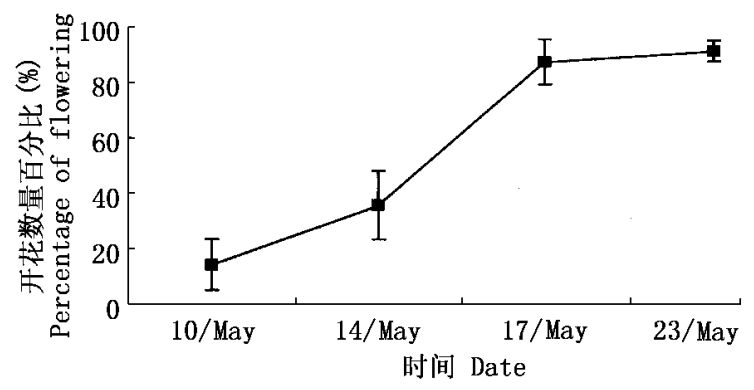

图 3 栲树的花期动态

Fig.3 Flowering dynamic of Castanopsis fargesii

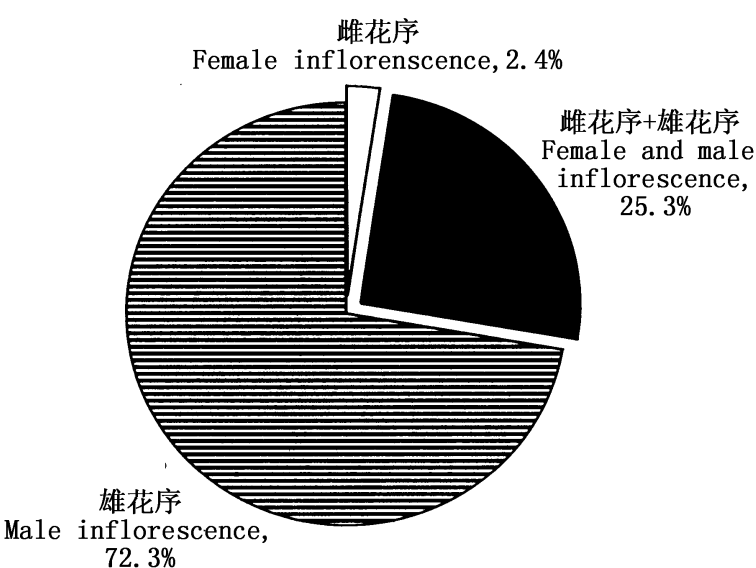

图 4 栲树雌花序和雄花序的比例

Fig.4 Percent of male and female inflorescence of Castanopsis fargesii

\section{2 雄花和雌花的数量比}

性比一般指同一种群内的雌雄个体的比值。同 株植株内的雌雄花的比值,也常作为性比进行研究 (陈家宽等,1994)。图 4 是栲树雄花序、雌花序数量 的比例。统计结果表明, 仅由雄花序组成的圆雉花 序所占比率最高, 而由雌花序组成的仅占 $2.4 \%$, 其 余是由雌花序和雄花序共同构成的圆雉花序。显
然 栲树雄花序的数量明显高于雌花序数量, 总体水 平上 雄花序约占花序总数的 $77.88 \%$,而雌花序仅 占 $22.12 \%$ 。从单个花序水平分析, 栲树的雄花数 量明显多于雌花数量, 每个雄性 $\square$ 荑花序上平均有 $(67.9 \pm 16.7)$ 朵小花,而每个雌花序上仅有雌花 $(26.10 \pm 5.08)$ 个 雄花与雌花的数量比约为 $9.2: 1$ 。

\section{3 花序的空间配置}

花序的生成与芽的分化有关。栲树花芽的分化 和发育主要集中在一级生殖枝上,约占整个的 $91.92 \%$ ，二级枝上有 $7.07 \%$ 的芽分化为花芽，在三 级枝上大约也有 $1.01 \%$ 的芽是花芽。但在栲树每 个生殖枝上，并非所有的芽都分化、萌发生成花序， 在图 5 中，顶芽萌发成花序的比例较高，约有 23 . $21 \%$ 侧芽的萌发也集中于生殖枝的上部，即远轴 端，主要包括第一侧芽、第二侧芽、第三侧芽及第四 侧芽等，第五侧芽及其以下各侧芽的分化成花序的 比例不到 $10 \%$ ，并向近轴端方向逐渐下降。

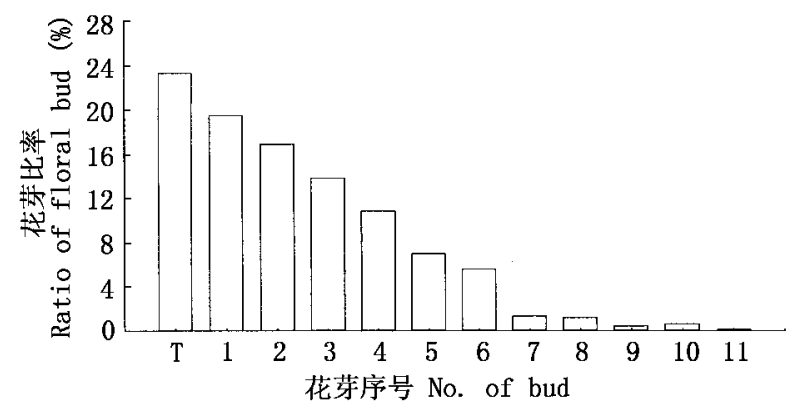

图 5 栲树不同生殖枝上花芽的分化

( $\mathrm{T}$ : 顶芽 ; 1 2 ‥是第一侧芽、第二侧芽 $\cdots$ )

Fig. 5 Percent of the different buds becoming inflorescence on the reproductive branch

( $\mathrm{T}$ : Terminal bud $1,2 \cdots$ the first lateral bud , the second lateral bud $\cdots$ )

栲树生殖构件在植冠层内不同方向的配置不同 (表 1)。同一植冠内向阳面生殖枝上芽萌发成花序 的比率有明显差异 $(p<0.01)$, 而且阳面生殖枝上顶 芽萌发成花序的比率与阴面生殖枝的比率也有显著 差异 $(p<0.05)$, 前者萌发成花序的比率平均为 $78 \% \pm 5.7 \%$,后者为 $67 \% \pm 5.7 \%$ ，但前三位侧芽的 萌发比率在同一植冠层的阳面和阴面没有差异 $(p$ $>0.05)$ 。以每个生殖枝为单位计算, 在同一植冠层 内 分布于阳面的每个生殖枝上的圆雉花序数多于 阴面, 两者差异明显 $(p<0.05)$ 。由前文得知, 每个 口荑花序上约有 $(67.9 \pm 16.7)$ 个小花, 则阳面每个 生殖枝上平均雄花数量约为 332 朵, 阴面的每个生 殖枝上约有雄花 258 朵, 阳面的生殖枝上的雄花数 量比阴面高 $28.7 \%$ 。 
表 1 栲树植冠层内不同方向的生殖特征

Table 1 The reproductive characteristic of Castanopsis fargesii within its crown

\begin{tabular}{|c|c|c|c|}
\hline & $\begin{array}{c}\text { 植冠向阳面 } \\
\text { Crown-toward-sun }\end{array}$ & $\begin{array}{c}\text { 植冠背阴面 } \\
\text { Crown-toward-shade }\end{array}$ & $\begin{array}{l}\text { 显著性1) } \\
\text { Significance }\end{array}$ \\
\hline 生殖枝上芽萌发成花序的比率 & $46.0 \pm 9.7$ & $36.7 \pm 7.7$ & $* *$ \\
\hline $\begin{array}{l}\text { Ratio of floral bud per reproductive branc } \\
\text { 顶芽萌发成花序的比率 }\end{array}$ & $78.0 \pm 5.7$ & $67.0 \pm 5.7$ & * \\
\hline $\begin{array}{l}\text { Ratio of terminal floral bud } \\
\text { 第一侧芽的萌发比率 }\end{array}$ & $84 \pm 7.01$ & $75 \pm 5.0$ & n.s. \\
\hline $\begin{array}{l}\text { Ratio of the first lateral floral bud } \\
\text { 第二侧芽的萌发比率 }\end{array}$ & $70 \pm 6.1$ & $71 \pm 10.8$ & n.s. \\
\hline $\begin{array}{l}\text { Ratio of the second lateral floral bud } \\
\text { 第三侧芽的萌发比率 }\end{array}$ & $75 \pm 11.7$ & $64 \pm 9.6$ & n.s. \\
\hline $\begin{array}{l}\text { Ratio of the third lateral bud } \\
\text { 生殖枝上的圆雉花序数 } \\
\text { No. of panicle per reproductive branch }\end{array}$ & $4.89 \pm 1.82$ & $3.80 \pm 2.08$ & * \\
\hline
\end{tabular}

1) 独立样本 $t$ 检验 Independent sample $t$-test $\quad$ n.s. : 不显著 Not significance $(p>0.05) \quad *: 0.01<p<0.05 \quad * *: p<0.01$

\section{3 讨 论}

\section{1 栲树的花期动态}

不同物种的花期不同, 在温带地区, 凤媒植物的 花期常发生在展叶期之前完成 (Rathcke \& Laqcey， 1985)。栲树是雌雄同株、风媒的口荑花序, 其花序 形成和开花时间在 4 月底至 5 月底间, 明显要晚于 展叶时间, 与叶片的生长时间重叠。风媒过程是个 相对被动的过程 (Passive process)，花粉的释放、散布 对生境条件特别是气候条件的要求相对较高, 其中 风被认为是一个主要因素, 其次温度和降雨也是影 响风媒植物授粉率的重要因素, 较低的湿度和降水 有利于风媒植物传粉的顺利进行( Whitehead ,1983)。 栲树在该地区 3 月底或 4 月初花芽开始萌动, 随着 气温的升高, 主要进行花序的生长发育, 花序要经 $25 \sim 30 \mathrm{~d}$ 的生长阶段; 盛花期集中于 5 月中旬至 5 月底间，此时约有 80\% 90\%的花开放, 相对于花 序的生长时间, 其花期和盛花期较短, 栲树在种群水 平上的花期持续约 $12 \mathrm{~d}$ 左右, 其盛花期在 $8 \mathrm{~d}$ 左右, 这段时间正是该地区温度逐渐回升、降雨明显较低 的时间，此时的气候状况有利于花粉的释放和散布。 在 5 月底和 6 月间因梅雨和台风的影响，该地区即 将到来第一个降水高峰(宋永昌等, 1995), 因此, 在 此之前完成花的发育和成熟, 有利于栲树有性生殖 过程的顺利进行，个体之间的花期虽有 $1 \sim 2 \mathrm{~d}$ 的差 异, 但仍相对集中, 即属于同步发生的花期 (Synchrony flowering），栲树种群的开花物候动态是对该 地区环境条件长期适应的结果。研究发现在某些雌 雄同株的植物中,一株个体上雄花和雌花的开花并 非同时发生，如 Pinus ponderosa、P. palustris、Quercus spp. 以及 Begonia spp. 等风媒植物, 在一个季节内， 雄花的开花时间要早于雌花的开花时间, 其生物学 适应意义在于降低自花受精的程度, 有助于增加不 同个体间异交的可能(Whitehead,1983; Willson,1983; Tapper ,1996)。由于 2000 年栲树雌花的数量较少， 观测、记录雌花花芽的分化、生长及其开花时间不完 整, 在 5 月中旬第一次发现雌花序, 此时每个雌花序 上约有 $22.6 \%$ 的雌花可见 3 个柱头。在栲树雄花序 的盛花期间, 每个雌花序上约有 $86.1 \%$ 的雌花可见 柱头, 在雄花的盛花期间多数雌花同时开放, 可能有 助于雌花较顺利地接受到花粉。从雄花序的盛花期 较短可以推测雌花发育和开花时间可能不会明显迟 于雄花, 否则雌花将无法接受到成熟的花粉, 完成受 精作用。

温度、降雨和光照也是影响该地区藤本植物春 芽活动的重要因素, 但栲树开花物候与该地藤本植 物明显不同, 藤本植物的开花盛期主要集中在 $5 \sim 6$ 月 结果盛期主要集中在 $8 \sim 9$ 月，与每年降雨量的 两个高峰相一致, 这种差异可能主要因为传粉机制 的不同, 因为该地区大多数藤本植物主要以虫媒为 主 (蔡永立 ,1998)。已有文献表明壳斗科中许多植 物种的花序释放出的精液味道对甲虫具有吸引力 (曹坤芳，1993）,Kaul(1986) 认为栲树的花序生长及 开花发生在早夏之际, 这个时期一般也是昆虫的活 动高峰, 因此推断栲树的花可能具有虫媒特征。从 本地区看 栲树盛花期间温度逐渐回升、降雨明显较 低，此时也是昆虫活动频繁的时间，笔者在野外实际 观测时, 发现有昆虫在栲树植株的花序上活动, 特别 是在光线较好的防火道两侧的栲树植株上, 中午时 分昆虫活动最为频繁。传粉方式是物种生殖过程中 
重要的进化特征，维持植物传粉系统的 3 个组成部 分是花粉、柱头和传粉媒介 (黄双全等,1999)，对栲 树而言, 其花部特征和传粉生物学特征仍有许多值 得研究的内容。

\section{2 栲树的雌雄花的数量比较和空间分布}

研究发现, 自然界中的风媒植物, 其雄花的数量 一般都远远超出雌花的数量 (Whitehead,1983)。本 文研究同时也表明栲树的雄花序数量明显多于雌花 序, 并且雄花的数量也明显多于雌花数量 二者之比 约为 9.2:1。栲树在春季通过大量的雄花及盛花期 的相对集中来散布大量的花粉, 以此保证雌花的受 粉和受精作用的完成, 是其有性生殖过程中的一个 重要对策, 这符合风媒植物的一般传粉特征。不少 壳斗科植物的种子产量在不同年份间具有较大的差 异 ( Silvertown \& Lovett Doust, 1993 ; Koenig et al. , 1994）其中风媒传粉假说认为具有风媒传粉特征的 植物在某些年份 (种子年) 可以产生大量的花和花 粉 花粉数量或浓度的增加会提高传粉有效性并使 受精和结实的比例增高，风媒种在种子年的异交水 平也很高(Smith et al.,1990; Koenig et al., 1994)。 对不少风媒物种的研究都支持这个假说, 如桦木属 的一些植物 (Betula spp.) , 它们的最大种子产量经 常在盛花的年份出现，即种子年，而非种子年中花的 数量则急剧减少 (Perala \& Alm, 1990) , 而且一些物 种在种子年时,干癔种子的比例明显较低 (Silvertown \& Lovett Doust，1993)。我们在两年的野外调查中发 现栲树雌花序的数量有差异, 栲树的种子产量也有 变化(陈波, 2001) , 但对栲树的雄花序和雄花仅进行 一个生长季的调查, 还无法断定雄花序或雄花的数 量在不同年份间有无明显变化, 如果有变化, 花粉可 能是限制受精及果实数量的一个因素; 如无明显变 化, 则可以推断尽管栲树雄花每年数量都很多, 但这 可能仅是栲树顺利完成有性生殖的一个必要条件， 生殖的最终结果一果实(种子)的发育、生长以及 数量的变化可能取决于雌花序或雌花的数量, 而果 实的进一步发育、生长以及种子的最终产量与植株 个体的资源积累和动物捕食压力有关。对雌雄异 株、风媒的欧洲白蜡树 (Fraxinus excelsior) 来讲, 其雌 株个体仅在种子年时产生盛花, 而雄株则几乎年年 持续开花并且数量变化不大, 从种子产量的年间变 化来看 花粉数量对其最终生殖结果的影响并不明 显(Tapper ,1996)。方炎明(1996)认为植物的雌性生 殖成分对花粉数量增加的反应呈现不同的规律, 如 果在生境资源限制植物结实的条件下 增加大量的
花粉只提高种子的质量而不是数量, 如果资源充足， 增加花粉可以提高种子数量而不一定提高种子质 量。

栲树花序和花的数量在植冠层内的配置明显不 同。分生组织最终分化成营养结构 (如枝、叶) 还是 生殖结构 (如花或花序), 在一定程度上也取决于枝 条在植冠内的位置以及分生组织在枝条上的位置 (Watson \& Casper ,1984) ，而且 植株的不同分枝格局 可以通过限制有性分生组织的数量或分化来影响有 性生殖的过程或结果（Tomlinson，1983; Preston， 1999)。栲树花芽的分化和发育主要集中在一级生 殖枝上 $(91.92 \%)$ :一方面, 由于一级枝上叶片数量 较多，因此光合作用生成的物质较多，可以保证有性 生殖过程的消耗; 另一方面，尽管栲树开花时间晚于 展叶时间, 但一级生殖枝主要位于植冠层外侧, 花粉 仍能顺利散布。在二级枝上芽的分化约 $7.07 \%$,三 级枝上仅大约 $1.01 \%$ 的芽可分化为花芽，由于在生 殖枝系中，二级枝至少在两年以上，因此，这也表明 栲树的花芽仍有少数是从休眠芽中来的。栲树植冠 内向阳面生殖枝上花序的比率与阴面生殖枝的比率 也有显著差异 $(p<0.05)$, 阳面生殖枝上的雄花芽萌 发成花序的比率有明显差异 $(p<0.01)$, 而且阳面生 殖枝上顶芽萌发成花数量比阴面高 $28.7 \%$ 。对栲 树个体而言, 树冠中上层阳面接受到的光照充足, 净 光合速率大，同化作用强，积累的营养物质多，因而 用于生殖的投资多于阴面, 这与对白桦(Betula platyphylla)的研究结果相似(祖元刚等, 2000)。栲树 花芽在植冠层中的配置表明, 花芽的分化除了与枝 系的分布有关 (具有明显的位置效应)，另一个适应 意义在于保证花粉的顺利散布和接受。

\section{参 考 文 献}

Brett, D.W. 1964. The inflorescence of Fagus and Castanea and the evolution of the cupules of the Fagaceae. New Phytologist, 63: $96 \sim 118$.

Borchert, B. \& N. A. Slade. 1981. Bifurcation ratios and the adaptive geometry of trees. Botanical Gazette, 142:394 401 .

Cai, Y.L. (蔡永立). 1998. Studies on vine ecology of the subtropical region in east China. Ph. D. dissertation of East China Normal University. (in Chinese with English abstract)

Cao, K. F. (曹坤芳). 1993. An overview of plant reproductive ecology. Chinese Bulletin of Botany (植物学通报), 10(2):15 23. (in Chinese with English abstract)

Chen, B. (陈波). 2001. Study on reproductive ecology of Castanopsis fargesii Franch. Ph. D. dissertation of East China Normal University. (in Chinese with English abstract)

Chen, J. K. (陈家宽) \& J. Yang (杨继). 1994. Plant evolutionary biology. Wuhan: Wuhan University Press.20. (in Chinese)

Dafni, D. 1992. Pollination ecology: a practical approach. Oxford: Oxford University Press. 
Ding, S. Y. (丁圣彦) . 1999. Comparative ecology of successive serial of evergreen broad-leaved forest. Kaifeng: Henan University Press. (in Chinese)

Fang, Y.M. (方炎明). 1996. Plant reproductive ecology. Ji’ nan: Shandong University Press. (in Chinese)

Fey, B.S. \& P.E. Endress. 1983. Development and morphological interpretation of the cupule in Fagaceae. Flora, 173:451 468 .

Harper, J. L. 1977. Population biology of plants. London: Academy Press.

Huang, S. Q. (黄双全)，Y. H. Guo(郭友好), M. Q. Pan(潘 明清) \& J.K. Chen(陈家宽) . 1999. Floral syndrome and insect pollination of Liriodendron chinense. Acta Botanica Sinica (植物学报), $41: 241 \sim 248$. (in Chinese with English abstract)

Kaul, R. B. 1985. Reproductive morphology of Quercus ( Fagaceae) . American Journal of Botany, 72:1962 1977.

Kaul, R. B. 1986. Evolution and reproductive biology of inflorescences in Lithocarpus, Castanopsis, Castanea and Quercus (Fagaceae). Annals of Missouri Botanical Garden, 73:284 296.

Koenig, W. D. , R. L. Mumme, W. J. Carmen \& M. T. Stanback. 1994. Acorn production by oaks in central costal California: variation within and among years. Ecology, 75:99 109 .

Lehtila, K., J. Tuomi \& M. Sulkinoja. 1994. Bud demography of the mountain birch Betula pubescens ssp. tortuosa near tree line. Ecology, $75: 945 \sim 955$.

Liu,Z. H. (刘智慧). 1990. A primary study on the structure and dynamics of Castanopsis fargesii population on Jinyun Mountain, Sichuan province. Acta Phytoecologica et Geobotanica Sinica(植 物生态学与地植物学学报), 14: 120 128. (in Chinese with English abstract)

Lovett Doust, J. \& L. Lovett Doust. 1988. Plant reproductive ecology: patterns and strategies. Oxford : Oxford University Press.

MacDonald, A. D. 1979. Inception of the cupule of Quercus macrocarpa and Fagus grandifolia. Canadian Journal of Botany, 57: $1777 \sim 1782$.

Maillette,L. 1987. Effects of bud demography and elongation patterns on Betula cordifolia near the tree line. Ecology, 68:1251 1261 .

Perala, D.A. \& A. A. Alm. 1990. Reproductive ecology of birch: a review. Forest Ecology Management, 32:1 38 .
Preston, K. A. 1999. Can plasticity compensate for architectural constraints on reproduction? Patterns of seed production and carbohydrate translocation in Perilla frutescens. Journal of Ecology, 89: $697 \sim 712$.

Rathcke, B. \& E. P. Laqcey. 1985. Phenological patterns of terrestrial plants. Annual Review of Ecology and Systematics, 16: $179 \sim 214$.

Silvertown, J. W. \& J. Lovett Doust. 1993. Introduction to plant population biology. Oxford: Blackwell Scientific Publications. $162 \sim 168$.

Smith, C. C., J. L. Hamrick \& C. L. Kramer. 1990. The advantage of mast years for wind pollination. American Naturalist, 136: $154 \sim 166$.

Song, Y.C. (宋永昌) \& X.R. Wang (王祥荣). 1995. Vegetation and flora of Tiantong National Forest Park, Zhejiang Province. Shanghai: Shanghai Science and Technology Press. (in Chinese)

Tapper, P.-G. 1996. Long term patterns of mast fruiting in Fraxinus excelsior. Ecology, 77:2567 2572 .

Tomlinson, P. B. 1983. Structure elements of the rain forest. In: Golley, F. B. ed. Tropical rain forest ecosystems: structure and function. Ecosystems of the world. 14A. Amsterdam: Elsevier.9 $\sim 28$.

Watson, M. A. \& B. B. Casper. 1984. Morphogenetic constraints on patterns of carbon distribution in plants. Annual Review of Ecology and Systematics, 15:235 258.

Whitehead, D.R.1983. Wind pollination: some ecological and evolutionary perspectives. In: Real, L. ed. Pollination biology. Orlando: Academic Press.

Willson, M. F. 1983. Plant reproductive ecology. New York: John Wiley \& Sons.

Zhong, Z. C. (钟章成). 1988. Ecological studies on evergreen broad-leaved forests. Chongqing: Southwest China Normal University Press. (in Chinese)

Zu，Y. G. (祖元刚)，Z.J. Mao(毛子军)，X. Y. Yuan (袁晓 颖) \& Y. X. Zhao (赵玉香). 2000. The blooming and production of reproductive modules in relation to tree age and their position within crowns in Betula platyphylla. Acta Ecologica Sinica (生态学报), 20:673 677. (in Chinese with English abstract) 